\title{
Towards an Immersive Learning Knowledge Tree - a Conceptual Framework for Mapping Knowledge and Tools in the Field
}

\author{
Dennis Beck \\ Curriculum \& Instruction \\ University of Arkansas \\ Fayetteville, U.S.A. \\ debeck@uark.edu \\ Christian Gütl \\ Technical University of Graz \\ Graz, Austria \\ c.guetl@tugraz.at \\ Scott Warren \\ University of North Texas \\ Denton, U.S.A. \\ Scott.Warren@unt.edu
}

\author{
Leonel Morgado \\ Universidade Aberta \& INESC TEC \\ Coimbra, Portugal \\ leonel.morgado@uab.pt \\ Andreas Dengel \\ Julius-Maximilians-Universität \\ Würzburg \\ Würzburg, Germany \\ andreas.dengel@uni-wuerzburg.de \\ Jonathon Richter \\ Immersive Learning Research Network \\ Missoula, U.S.A. \\ jonathon@immersivelrn.org
}

\author{
Mark Lee \\ Charts Sturt University \\ Albury-Wodonga, Australia \\ mark@immersivelrn.org \\ Minjuan Wang \\ School of Journalism \& Media Studies \\ San Diego State University \\ San Diego, U.S.A. \\ mwang@sdsu.edu
}

\begin{abstract}
The interdisciplinary field of immersive learning research is scattered. Combining efforts for better exploration of this field from the different disciplines requires researchers to communicate and coordinate effectively. We call upon the community of immersive learning researchers for planting the Knowledge Tree of Immersive Learning Research, a proposal for a systematization effort for this field, combining both scholarly and practical knowledge, cultivating a robust and ever-growing knowledge base and methodological toolbox for immersive learning. This endeavor aims at promoting evidence-informed practice and guiding future research in the field. This paper contributes with the rationale for three objectives: 1) Developing common scientific terminology amidst the community of researchers; 2) Cultivating a common understanding of methodology, and 3) Advancing common use of theoretical approaches, frameworks, and models.
\end{abstract}

Index terms-immersive learning, Knowledge Tree, research, epistemology, ontology

\section{INTRODUCTION}

The field of immersive learning research is heavily interdisciplinary and thus traveled by a scattered community of researchers, who journey through it from different areas of knowledge. These include: Educational technology, computer science, game design, learning sciences, psychology, biomedical sciences, narrative studies, arts, design, media studies, communication sciences, and the thousands of disciplinary and occupational content areas wherein immersive learning and training may be relevant. Each discipline carries its own history of knowledge development and brings its related assumptions, theories, and practices into the field of immersive learning. Wondering may be the cornerstone of knowledge development and wandering the means to delve into its farthest reaches. Yet, combining efforts for better exploration of this field and its topography from the different disciplines does require wanderers to communicate and coordinate effectively, developing a joint perspective of the field and devising the means to blaze trails. Wanderers may climb a tree to attain that perspective and come up with those means, and as such we call upon the community of immersive learning researchers for planting such a Knowledge Tree, supporting research wanderers through the field.

Growing such a tree would involve developing common terminology amidst the community of researchers, and a common language - or at least mutually intelligible languages, bringing together diverse areas of research. Currently, the field of immersive learning is a Babel of unintelligibility, with strikingly different scope for terms as foundational for its meaning as "immersion", as analysis of the thin theoretical grounding of recent surveys in the field demonstrates [1]. The perspective of the field status is muddled, the current knowledge partially disjointed, specifically among different disciplines. As a result, researchers who are wandering the field struggle to collaborate with others.

The Knowledge Tree of Immersive Learning Research, a proposal presented herein, is thus a systematization effort for this field, integrating both scholarly and practical knowledge. Its purpose is to cultivate a robust and ever-growing knowledge base and methodological toolbox for immersive learning, aimed at promoting evidence-informed practice and guiding future research in the field.

The main contribution of this paper focuses on three objectives: 1) Developing common scientific terminology 
amidst the community of researchers; 2) Cultivating a common understanding of methodology, and 3) Advancing common use of theoretical approaches, frameworks, and models. Thus, the remainder of the paper is structured as follows: Background and related work, Steps taken toward development of the Knowledge Tree, and a First Concept of the Knowledge Tree of Immersive Learning Research.

\section{BACKGROUND AND RELATED WORK}

\section{A. The Concept of Immersion}

Many scholars assume an intuitive understanding of the concept of "immersion", without even citing a definition [1]. This intuitive understanding was originally expressed by Murray's analogy to being submerged in water, a "sensation of being surrounded by a completely other reality" [2]. Scholars across technology-centered fields of study have adopted this perspective and developed it into two main theoretical views, both employed by diverse groups of authors. The first is the concept of immersion as an attribute, quality, or characteristic of a technological system [3]. The second view focuses on the participants' response to being surrounded or submerged [4]. However, outside the field of technology, and prior to its preoccupation with immersion, other fields have been discussing this topic: learning sciences, psychology, literature studies, the arts, etc. These fields discussed how narratives, engagement, psychological flow and other factors have contributed to immersion as a phenomenon [5]. Over 20 years have passed since the two technological-centered theoretical views debuted, yet the scholarly activity in the field of immersive learning research has neither embraced a definition nor combined it with the concurrent non-technological views. In the field of cognitive science, Slater may have come the closest by acknowledging the role of narrative, not just the technology attributes [3]. This fragmented perspective on immersion is reflected in the current literature reviews published in the field, with many of them simply selecting a definition without explanation, evidence, or critical appraisal [1], often intertwined or synonymous with other concepts such as presence, involvement, flow and engagement [5]. This has short-circuited the impact of the reviews as they have not been successful in bridging theoretical perspectives on immersion with current research results, and thus have not been able to highlight the gap in the existing research: Many do not even cite other previous reviews [6].

More recently there have been significant efforts to develop a comprehensive definition of immersion. The research team of Nilsson and colleagues from Denmark performed an exhaustive, interdisciplinary review of the definitions of immersion in order to develop a three-dimensional taxonomy, which they illustrate with the "immersion cube" diagram [5]. The immersion cube provides a theoretical structure for understanding immersion in three different dimensions: system immersion, narrative immersion, and challenge-based immersion. The experienced immersion is thus understood as the nexus of these dimensions. The cube can be used to measure and visually display an interpretation of immersion as varying levels of the three dimensions. The system immersion dimension reflects the properties of the system, which comprises Slater's definition [9] and simultaneously highlights it as unidimensional. The narrative immersion dimension reflects the "degree of mental absorption or intense preoccupation with the story, the diegetic space, and the characters inhabiting this space" (id., p. 114). This highlights content-based immersion (system and narrative) as bidimensional. Finally, challenge-based immersion reflects "a user's mental absorption brought about by the experience of challenges requiring mental or sensorimotor skills" (p. 116). This extrudes the agency of the immersed as an essential dimension of the immersion phenomenon.

These dimensions comprise the diversity of other aspects employed in the field. For instance, challenge-based immersion includes aspects such as what Adams [7] described as strategic immersion (optimization of choices, rather than meaning, e.g., focusing on winning a game rather than following the storyline) and tactical immersion, which occurs when attention is absorbed reacting to obstacles or enjoyment - an interpretation which is extremely like Csikszentmihalyi's concept of flow [8]. Presence is another major parallel concept related to immersion. Described as the feeling of "being there" [8], this psychological state is more extensive than that. Alternative views include Lombard and Ditton's [9] definition of presence as the perception of non-mediation, Biocca's [10] contribution of presence as arising from a mental imagery space, Slater [11] and Waterworth \& Waterworth's attentional perspective on presence as emphasis on perception of stimuli [12], and Riva et al.'s [13] view of presence as a biological and cultural mechanic for selfmaking sense of sensorial input. Nilsson et al. [5] discussed how these four views on presence are related to the three dimensions of system, narrative, and challenge-based immersion. Slater's emphasis on measurability of presence maps to the concept of system immersion, for instance; the self-making from sensory input is related to the combined dimensions of challenge-based immersion and narrative immersion. The three-dimensional view of immersion integrates these diverse perspectives on presence, from an alternative theoretical viewpoint.

Although Nillson and colleagues provided this clarity towards our understanding of immersion, they did not define it. That pragmatic contribution was provided by Agrawal, Simon, and Bech [14], who although without being aware of Nillson et al.'s work did reach an identical conclusion from the extant literature and synthesized it as "a phenomenon experienced by an individual when (...) in a state of deep mental involvement in which (...) cognitive processes (...) cause a shift in (...) attentional state such that one may experience disassociation from the awareness of the physical world" (p. 5). Their definition thus supplies a practical component to complement the immersion cube taxonomic framework. Thus, the ontological roots of the Knowledge Tree include both Agrawal et al.'s definition and Nilsson et al.'s immersion cube.

\section{B. The Concept of Immersive Learning}

Combining the concept of learning with the above discussed concept of immersion broadens the focus of our topic, thus increasing the number and angles of viewpoints, as well as introducing other disciplinary perspectives. Thus, in our notion of the knowledge tree, new branches open paths to be considered and influence an overall understanding.

Learning is currently seen as a phenomenon, occurring amidst a variety of contexts. This holistic view considers social, 
cultural, cognitive, and biological contexts as providing relevant perspective and insights to the understanding of the phenomenon of learning [15]. Learning research addresses both focused topics (such as individual and topic particularities, processes and their design and content) and wider contextual impacts (motivation, changes brought about by technology, lifelong learning, regulatory aspects).

With this understanding of learning, immersion is introduced as a lens and instrument to investigate, understand, and manipulate those contexts [1]. From the holistic view on learning, it follows, for instance, that immersive learning addresses not only the learning outcomes, but also the mutual relationship between the provided educational medium, the learners' perception and cognitive processes as well as their motivational and affective states and traits [16], among other topics, including organizational and social aspects. Researchers on immersive learning may consider utilizing immersion as both a method to understand a topic and as a means of exploring its impacts on specific learning variables. For example, researchers may explore the role of culture in learning and development by focusing on how various aspects of culture can factor into the experience of a state of deep mental involvement for learners, via culture's systemic, narrative, and challenge-based aspects. Conversely, other researchers may be interested in the impacts of specific system, narrative, and challenge-based immersion variables on prenatal and lifelong brain development and maturation. Others yet may apply "immersion" as a lens for teacher education, exploring how we can improve teacher education experiences through the manipulation of system properties, the learner agency (challenge-based dimension), and narrative components.

\section{Problem and Purpose}

As addressed above, the main issue identified is that the field of immersive learning research is fragmented due to its interdisciplinary nature and diverse community of researchers. The disjointed landscape of immersive learning research features multiple literature reviews that do not use common definitions of important terms or methods, and a lack of citing of each other's work. All of this has resulted in a weak theoretical grounding for the field of immersive learning research, a lack of clear directions for the future, and little collaboration across academic disciplines.

To overcome the problem stated above, we propose a Conceptual Framework for building a common and agreed understanding as well as for mapping knowledge, tools, and services in the field. The proposed "Knowledge Tree of Immersive Learning Research" or "Immersive Learning Knowledge Tree" / "iLRN Knowledge Tree" aims for a systematization effort for this field, combining both scholarly and practical knowledge. Its purpose is to cultivate a robust and ever-growing knowledge base and methodological toolbox for immersive learning, to promote evidence-informed practice and guiding future research related to immersive learning.

Towards the steps to build the iLRN Knowledge Tree and maintain its value for the broader community, scholarly and practical aspects and viewpoints must be considered. Thus, it requires researchers to include and synthesize a broad body of scholarly sources, such as (a) Scoping and systematic reviews on a diverse range of immersive learning constructs and concepts; (b) ontology(ies) and related taxonomies for capturing empirical research findings on the relationships between the constructs identified in the scoping reviews and synthesis of findings; (c) a conceptual model to encourage channeling of future research efforts and contributions across the field toward a shared overall framework and agenda. However, practical knowledge sources are also important for the systemization of an applied field such as immersive learning. This will include practical sources such as (a) an evidence repository, based on the taxonomic framework and conceptual data model, enabling the derivation of evidence-based design principles, guidelines, and best practices for engaging, effective and efficient learning experiences; (b) community-curated collection of exemplars embodying and demonstrating the operationalization of the principles, guidelines, and best practices; (c) guidelines and references to resources - theoretical foundations, practical examples and existing tools and services - to assist educators and developers in their implementation.

\section{STEPS TAKEN TOWARDS THE DEVELOPMENT OF THE IMMERSIVE LEARNING KNOWLEDGE TREE}

Towards the realization of the Immersive Learning Knowledge Tree, the researchers of the iLRN community have paved the way and provided soil and seeds for building the envisioned conceptual framework.

\section{A. Development of Methods}

1) Scoping Review Protocol

Our initial consideration was to develop methods for authoring systematic literature reviews $[1 ; 6]$. These focus on the identification and retrieval of scholarly literature that answers a specific question to inform practice, policy, and further research - a critical instrument for developing a joint vision of the field of immersive learning. However, we quickly discovered that the undeveloped and fragmented status of the field of immersive learning would render systematic review efforts unfeasible for matching against each other. The building of a common understanding needed more of a groundwork approach, one that scoping reviews would provide. Thus, we developed a protocol for doing scoping reviews on immersive learning, enabling the community to identify knowledge gaps, identify the location and scope of evidence on the body of immersive learning literature [1]. We also viewed this scoping review protocol as a means for uniting the methods in the field under one banner. Its use is underway and hopefully will provide an exemplar of the importance of common concepts and criteria for future, more specific, systematic literature reviews.

\section{2) Inter-rater Vetting Process for Immersion Cube Classification \\ Our first use of the scoping review protocol was to explore} the actual research-based accounts of educational uses, practices, and strategies in immersive learning. This priority stems from a key challenge faced by earlier generations of research on technology-influenced areas of education: a tunnelvision focus, perhaps born of enthusiasm, to attempt to ascertain direct impacts of technology into particular aspects of learning. Inevitably, each generation of educational technology, from Pressey's teaching machines [17] to modern extended reality, through computer-aided learning, edutainment, serious games, 
and others, went through this, reaching the inevitable conclusion: learning does not depend solely on the technology, but on its overall context and method of use; and it is not neutral nor unidirectional, since technology both changes and is changed by its adoption. This, which Papert criticized over three decades ago as technocentric research [18], has been the realization of information systems research in general, which has focused on the need for multidimensional analysis for understanding and influencing technology-infused phenomena [19]. To break out of the tunnel vision of spurious techno-centric research, the community needs a perspective on the available contextual knowledge: the actual uses, practices, and strategies taking place with immersive learning. This application of the scoping review protocol implied conducting a survey of surveys of this field, which has already yielded a panorama on the uses of immersive learning and the current gaps [6], as mentioned in the next subsection.

In pursuing this survey of surveys, the question arose on how to situate uses of immersive learning within the theoretical perspective of immersion. The immersion cube mentioned above provided a framework, but not a method. For this, we established an inter-rater vetting process for classification amidst the immersion cube. Inter-rated vetting compares and contrasts researchers' independent classifications to minimize bias [20]. For immersive learning uses, our method had researchers independently consider each unit under scrutiny on system immersion, narrative immersion, and challenge immersion, classifying them at 0.25 intervals between 0 and 1 representing a qualitative 5-point Likert scale from no association to full association with each immersion dimension, followed by an interrater process where the panel of researchers discussed these classifications until reaching consensus [6].

3) Validated Questionnaire on Technological Research Priorities for Immersive Learning

To identify technological research priorities on immersive learning we decided it is necessary to survey the community of immersive learning researchers. To do this, we authored a questionnaire [21] designed to gather the researchers' priorities regarding the challenges hampering widespread adoption of immersive environments in learning contexts, focusing on several issues within access, content production, and deployment. Thus, grounded in the literature on these topics, we conducted an expert validation process of the questionnaire that involved three cycles of feedback and revision. This process provided important recommendations on the structural, context clarification, ambiguity, and missing aspects of the questionnaire. We then deployed it with the researchers involved with events organized by the Immersive Learning Research network, resulting in a ranking of these research priorities [24].

\section{B. Application of Methods to Scholarship}

\section{1) Scoping review of survey of surveys}

The application of the methodological contributions described in the previous subsection has yielded early seeds of the Knowledge Tree effort. Applying the scoping reviewing protocol methodology, which dictates a survey of surveys to demonstrate the gap in current knowledge, resulted in a rhizomatic view of the cohesion of the field. Out of 47 identified surveys between 2014 and 2019, only 29 of those cited at least one of the others. The other 18 (i.e., over a third) were outliers, that did not cite and were not cited by other surveys [6]. Further, those 29 mostly acknowledge a single survey from 2017, which was specifically about augmented reality in education [22] and only cited one of the earlier surveys, also about this same topic [23]. Thus, the field of immersive learning research is scattered in what should be its very source of cohesion: its surveys of the literature. The augmented reality systems approach to immersion has some cohesion, but not the field of immersive learning itself.

\section{2) Operationalizing the Immersion Cube}

A second result of this survey of surveys is an examination of actual uses of immersive learning environments, applying the inter-rater vetting process for classification amidst the immersion cube. This spatial positioning and dimensioning within the immersion cube revealed six major clusters of uses (Complementing, Simulating, Exploring, Engaging, Experiencing, and Accessing). The spatial aspects of these clusters within the immersion cube also revealed four voids to current research on uses of immersive learning: Low immersion uses, challenge-focused immersion uses, low-tech immersion uses, and combining high-tech with strong interdisciplinary aspects [6]. The clusters highlighted current pathways for research, and the void both inspired new pathways and indicated how some lines of inquiry are occurring outside the theoretical lens of immersion. This survey of surveys effort is still undergoing analysis of its results, with an analysis of the educational practices and strategies underway.

\section{3) Utilizing the Technological Research Priorities} Questionnaire with the Immersive Learning Community

In parallel, we applied the technological research priorities questionnaire, by surveying the immersive learning research Network (iLRN) community of researchers on their priorities for technology research. Defining this community as researchers involved with iLRN-organized events (committee members, organizers, keynote speaker, and authors), the results ranked and clustered 43 priorities, with the topmost being "Creating/identifying solutions for teachers/trainers to be able to identify learning support needs and provide extra resources directly within immersive environments" [24].

\section{INTRODUCING A FIRST CONCEPT OF THE IMMERSIVE LEARNING KNOWLEDGE TREE}

Patterned after a natural tree, the Immersive Learning Knowledge Tree metaphor possesses soil, roots, trunk, branches, leaves, and even birds (Fig. 1). The roots, born of seeds of interest and inquiry, are the common definitions, methods, and research instruments. Growing from their initial energy drivers, they provide stability to the soil and then sap their sustenance from it. The soil here is the combination of ontologies, taxonomies and conceptual data models that become more consolidated as the roots develop, and then provide them sustenance for further growth. Roots and soil provide a strong foundation for the advancement of the field of immersive learning. The trunk represents the structural knowledge which surfaces from the ground and roots. This structural knowledge will be composed of scoping and systematic literature reviews on various topics related to immersive learning, of classified collections of facts like expert input from the field of immersive 


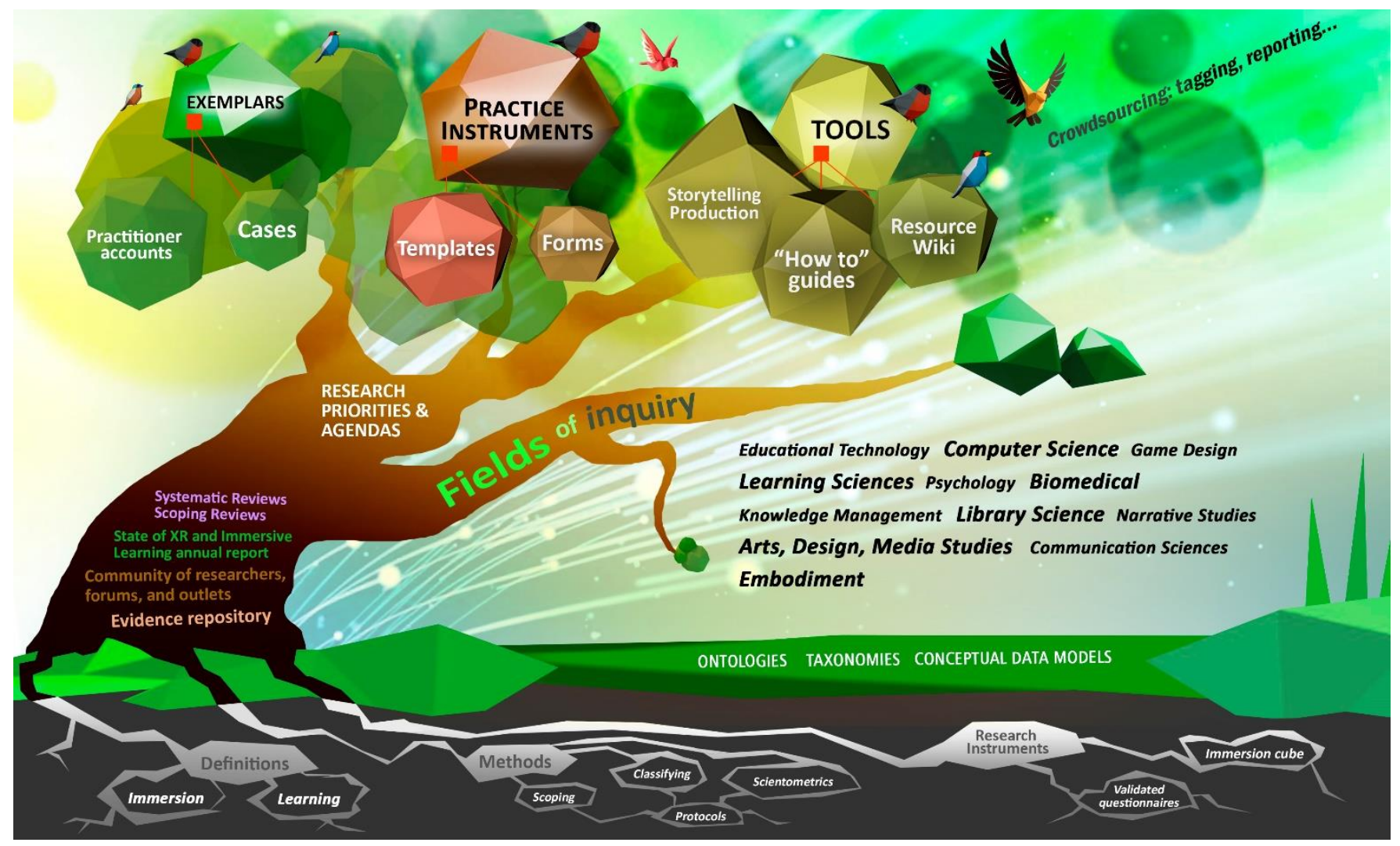

Fig. 1 - The Immersive Learning Knowledge Tree (original art by Demetrius Lacet).

learning (e.g., the iLRN State of XR and Immersive Learning Report) and evidence repositories; and of the awareness of the networked knowledge of the community researching immersive learning provided by Scientometrics. The branches of the knowledge tree, sprouting from the trunk, consist of two things: 1) The fields of inquiry which extend from the core trunk of immersive learning, leading to specific outcomes; and 2) the research priorities and agendas, intertwined with the fields of inquiry. The branches produce shoots which develop first leaf buds and then leaves, in this case in the form of exemplars (e.g., practitioner accounts, cases, software), instruments (templates for practice and research), and tools. The birds, here the community of immersive learning researchers and practitioners, employ these exemplars in their work (e.g., building nests), as well grouping some of the resources together through crowdsourcing efforts like tagging and reporting. But why cultivate such a tree? The next section explores our rationale.

\section{A. Knowledge Tree Rationale}

Due to the 'publish or perish' nature of academia, researchers often focus on short term research studies that are disconnected from an overall framework or agenda. This is unfortunate, because when individual efforts build upon each other, arguments can be contrasted, fact-checking can more readily be accomplished, and informed discussion ensue. An interdisciplinary field such as immersive learning adds additional complexity to this problem as scholars from different academic fields often approach their research using extremely different methods and terminology. Unfortunately, this has resulted in a feeble theoretical grounding for the field which has hindered its advancement, made it vulnerable to criticism, and suppressed interdisciplinary collaboration efforts.

The solution to our problem is of course the systematization of the field of immersive learning, and that begins with our understanding of its ontology - what is the nature and existence of what we are studying? This can aid researchers in identifying their level of confidence regarding what they are studying in terms of its nature and existence. For example, who decides what is 'real' or not in research? How do differing perspectives on reality help scholars better understand their research?

Emerging from the field's ontology is how we frame our research to discover new knowledge - epistemology. More specifically, how do we explore the scope, methods and validity related to the acquisition of knowledge? In the context of immersive learning environments, important questions to consider would be a) what constitutes a knowledge claim; b) what the process of knowledge acquisition is; and c) how we can measure transferability of knowledge. Epistemology is important because it influences how we frame our research in our attempts to discover knowledge. The interdisciplinary nature of immersive learning places challenges to developing such solid groundings, providing a way to overcome them is the driver of the Immersive Learning Knowledge Tree proposal. 


\section{B. The Roots - the Common Language (Definition, Methods, Instruments)}

The roots of the Immersive Learning Knowledge Tree are the common definitions, methods and research instruments that enter the ontological and epistemological soil and consolidate it as a strong foundation for the advancement of the field of immersive learning. As mentioned above, we need to agree as a field on common definitions for foundational scientific terminology such as immersion and learning, and to their combination on the phenomenon of immersive learning. The use of common definitions will enable the use of meta-analysis techniques, which help boost the power to study causality, by combining primary studies and providing a precise estimate of impacts and effects. Beginning at a similar starting point (e.g., common definitions) will also facilitate establishing professional collaborations with others in our diverse field.

Also, while preserving our interdisciplinary strengths, the immersive learning research community needs to coalesce around common methodological underpinnings, as well as begin to construct open instruments. These similar methods should be constructed for the most basic of research tasks such as scoping and systematic review protocols, and experimental and quasiexperimental research protocols, while allowing for the diversity of methodological approaches from the interdisciplinary fields of immersive learning to coordinate from these foundational methods. Again, starting at the same basis methodologically will help advance the field and make the use of scoping and systematic literature reviews much more impactful.

\section{The Soil - the Ontological and Epistemological Terrain}

Due to the interdisciplinary nature of the field, the soil of the Immersive Learning Knowledge Tree will be composed from a diverse collection of ontological knowledge: ontologies, taxonomies, and conceptual data models. These constructs will provide the essential ingredients necessary to pull together the disparate, fragmented research in the field into a comprehensive and comprehensible form. The ontological and epistemological soil will also provide guidance for scholars not only on how to frame their research in different ways, but also in how to use differing perspectives on reality as a lens for understanding and applying their research. This currently loose soil will be consolidated as methodological roots embed themselves within it and together soil and roots provide the foundation for the trunk of the Knowledge Tree.

\section{The Trunk - the Knowledge Growing More Solid Yearly}

The trunk of the Immersive Learning Knowledge Tree represents the structural knowledge which grows from the ontological and epistemological soil. Currently a sapling vying to become a gigantic sequoia, the trunk will be its solidity, its pillar and common strength. Its interconnected wood rings, sapping the roots, form scoping and systematic literature reviews on various topics related to immersive learning; classified collections of facts like expert input from the field of immersive learning (e.g., the iLRN State of XR and Immersive Learning Report) and evidence repositories; and the Scientometric awareness of the networked knowledge of the community researching immersive learning.
The scoping reviews will enlarge the trunk by identifying gaps in the scholarly knowledge, better understanding the depth and breadth of the literature, and clarifying specific concepts; the systematic reviews will further widen the trunk with focused answers from the literature to inform practice, policy, and future research - both to further strengthen the trunk and for its offshooting branches, twigs, and leaves.

The classification efforts lead to a repository (or mutually intelligible repositories) consisting of a collection of seminal research publications, empirical case studies, and open data sets. The classification efforts also lead and develop from expert input such as the State of XR and Immersive Learning annual report, a "multi-sector, cross-disciplinary initiative aimed at regularly surveying the XR and immersive learning landscape to identify the technological, pedagogical, and other innovations exhibiting the most promise, along with the major opportunities and challenges related to their uptake, adoption, and implementation; and advancing research and promoting research-grounded practice in the use of XR and immersive technologies for supporting learners across the full span of learning from K12 through higher education as well as in workplace, community, and lifelong learning" [25]. The diverse pieces of evidence within the repository and from expert input are linked to the knowledge provided by scoping and systematic reviews, providing a strong connection from which both further research and practice can branch out. This strong connection will also help to extend the impact of the scoping and systematic literature reviews through the authoring of evidence-based design principles, guidelines, and best practices encapsulating "what works" (and what does not) for creating effective and engaging immersive learning experiences.

The Scientometrics describing the network topology of the community researching immersive learning aim to build selfawareness among the community: who its members are, where they assemble to discuss and where they publish, which aspects of inquiry are being pursued, which citation networks reveal influences, trends, discussions, and interpretation, which topics. This information thus renders the community more cohesive, streamlining and supporting the academic discussion and exchange of viewpoints, and acting as the bonding element that strengthens the trunk matter.

Emerging from the epistemological roots and ontological ground, the scoping and systematic literature reviews on various topics related to immersive learning; classified collections of facts like expert input from the field of immersive learning and evidence repositories; and the Scientometric awareness of the networked knowledge of the community researching immersive learning all combine to form the trunk of the Immersive Learning Knowledge Tree.

\section{E. Branches - Knowledge Reaching Out}

The branches of the knowledge tree, sprouting from the trunk, consist of two things: 1) The fields of inquiry which extend from the core trunk of immersive learning, leading to specific outcomes; and 2) the research priorities and agendas of the field, intertwined with the fields of inquiry. The academic fields of inquiry include educational technology, computer science, game design, learning sciences, psychology, biomedical sciences, narrative studies, arts, design, media 
studies, communication sciences, and the thousands of other disciplinary and occupational content areas where immersive learning and training may be relevant.

This sprouting occurs from the trunk of scoping and systematic literature reviews, expert input, evidence repository, and community of research. For example, a systematic review of the uses of immersive learning environments with individuals with autism to advance their knowledge of STEM subjects is organically extending branches at least to the disciplinary fields of didactics (of science, technology, engineering, and mathematics), educational science, cognitive science, and communication science. An obstacle to immersive learning listed in the State of XR and Immersive Learning report [25] such as "interoperability" would branch out to different disciplinary approaches to it, from computer science and information systems to operations research and educational sciences. Researchers from a field such as English Literature studying narrative immersion would travel along the branches of their own field but would also leverage the awareness of the network of research to cross over to branches in other fields, such as computational content analysis or media studies, to discuss their views and findings with similarly interested immersive learning researchers.

\section{F. Leaves - the Practical Endeavors}

The leaves of the knowledge tree comprise exemplars (e.g., practitioner accounts, cases), instruments (templates for practice and research, forms), and tools. The 'tool' leaves may include a virtual choreography production tool, a resource creation wiki, and "How To" guides (e.g., how to apply a method), among many other forms. Leaves can be considered as the periphery of the structural knowledge branches (the fields of inquiry and research priorities). These rather practical endeavors should guide and support investigators in carrying out and situating their own research and practice within the immersive learning community.

\section{G. Birds - the Community}

Just as birds fly from branch to branch and leaf to leaf, finding nourishment and building nests, so the community of immersive learning researchers and practitioners employ exemplars (leaves) in their nest-building work (e.g., professional practice). For example, primary school history teachers interested in the use of augmented reality for a field trip may scan the various leaves of the Knowledge Tree for useful resources in their teaching, gathering them into lesson plans on the historical accomplishments of well-known figures. They may use virtual choreography production tools to keep records of the lessons or produce content for them, as well as author a "teachers' guide" to go with the lesson plans. As they do this, they can be encouraged to create common tags for these resources and share their self-authored resources so that future instructors may locate them as a well-curated grouping. Another example may be a researcher of technical immersion uses with individuals with autism, who may download the scoping review protocol to better understand the gaps in the research literature in the field and better situate one's own research within a gap. The researcher can also be encouraged to tag the completed scoping review and link it to the Knowledge Tree, becoming part of the trunk.

\section{WORK IN Progress AND FUtURE GROWTH OPPORTUNITIES FOR THE IMMERSIVE LEARNING KNOWLEDGE TREE}

Current work focuses on cultivating the roots and enriching the soil of the Immersive Learning Knowledge Tree. A working group of scholars have been meeting regularly to determine the scope, method and human resources needed for authoring the ontological and epistemological structure. Our hope is to present our progress at the 2021 Immersive Learning Research Network Conference. In parallel, we are developing a Scientometrics view of the field and community of immersive learning research for the purpose of standardizing, collecting, analyzing, and reporting data on a wide range of scholarly and practical activities to provide a clearer picture of our community's work. Our hope is that the regular reporting and dissemination of this data will build self-awareness among the community and thus bring us closer together, streamlining and supporting the academic discussion and exchange of viewpoints, and acting as the bonding element that strengthens the trunk matter. Another part of the root structure is common methods. To address this, we are also developing a rigorous, replicable method for classification of specimens of immersive learning aspects within the dimensions of the immersion cube. The current classification method [6] is rather rudimentary, a score-based method carried out by independent researchers with good scale-reliabilities, but an evidence-based scoring guideline is missing and must be pursued so that the comparison of methods, contributions, and gaps can be more comparable among the community.

Also, some of the authors of this paper are completing a survey of surveys of research on educational strategies and practices with immersive learning environments, which will provide another early contribution to the trunk of the Knowledge Tree. Also adding soon to the trunk will be the initial set of expert input, the first State of XR and Immersive Learning report to be published in March 2021, providing identification of technological, pedagogical, and other innovations in the field, along with the major opportunities and challenges related to their adoption and implementation. Our plan is to perform a comprehensive scoping review of the field on educational uses, strategies and practices with immersive learning environments, which will also add to the trunk of the Knowledge Tree, and to disseminate a call for proposals for scoping and systematic literature reviews on a diverse array of topics that will also strengthen and broaden the Knowledge Tree trunk.

\section{CONCLUSION}

The main contribution of this paper is providing a rationale for three objectives: 1) Developing common scientific terminology amidst the community of researchers; 2) Cultivating a common understanding of methodology, and 3) Advancing common use of theoretical approaches, frameworks, and models. As stated above, the perspective of the field of immersive learning research is in a state of confusion, with current knowledge partially disjointed, specifically among different disciplines. As a result, researchers who are exploring the field struggle to combine their efforts with others. The Knowledge Tree concept is an initial effort at bringing together the researchers and knowledge involved in this broad and diverse discipline, and just as in the cultivation of a real tree, future efforts will be needed to clarify concepts, prune 
ineffective approaches, and fertilize new opportunities for growth. We have provided a framework for systematizing this field, enabling to combine the efforts of scholars and practitioners. By articulating the various aspects of this framework with the metaphor of a tree, soil, and birds, we hope to have established conditions - seeding, as it may - for development of a robust knowledge base and methodological toolbox for immersive learning. Its aims are to promote evidence-informed practice and guide future research in the field.

\section{ACKNOWLEDGMENTS}

We would like to thank Demetrius Lacet for his design work on the Immersive Learning Knowledge Tree diagram.

\section{REFERENCES}

[1] L. Morgado and D. Beck, "Unifying Protocols for Conducting Systematic Scoping Reviews with Application to Immersive Learning Research," in 2020 6th International Conference of the Immersive Learning Research Network (iLRN), San Luis Obispo, CA, USA, Jun. 2020, pp. 155-162, doi: 10.23919/iLRN47897.2020.9155093.

[2] J. H. Murray, Hamlet on the holodeck: the future of narrative in cyberspace. New York, NY: Free Press, 1997.

[3] M. Slater, "Place illusion and plausibility can lead to realistic behaviour in immersive virtual environments," Philos. Trans. R. Soc. B Biol. Sci., vol. 364, no. 1535, pp. 3549-3557, Dec. 2009, doi: 10.1098/rstb.2009.0138.

[4] B. G. Witmer and M. J. Singer, "Measuring Presence in Virtual Environments: A Presence Questionnaire," Presence Teleoperators Virtual Environ., vol. 7, no. 3, pp. 225-240, Jun. 1998, doi: $10.1162 / 105474698565686$.

[5] N. C. Nilsson, R. Nordahl, and S. Serafin, "Immersion Revisited: A review of existing definitions of immersion and their relation to different theories of presence," Hum. Technol., vol. 12, no. 2, pp. 108-134, Nov. 2016, doi: 10.17011/ht/urn.201611174652.

[6] D. Beck, L. Morgado, and P. O'Shea, "Finding the Gaps about Uses of Immersive Learning Environments: A Survey of Surveys," J. Univers. Comput. Sci., vol. 26, no. 8, pp. 1043-1073, 2020.

[7] E. Adams, Fundamentals of game design, Third edition. Berkeley, CA: New Riders, 2014.

[8] M. Csikszentmihalyi, Flow: the psychology of happiness, Reprinted. London: Rider, 1997.

[9] M. Lombard and T. Ditton, "At the Heart of It All: The Concept of Presence," J. Comput.-Mediat. Commun., vol. 3, no. 2.

[10] F. Biocca, "Cyborg's Dilemma: Progressive Embodiment in Virtual Environments," J. Comput.-Mediat. Commun., vol. 3, no. 2.

[11] M. Slater, "A note on presence terminology," Presence Connect, vol. 3, no. 3, pp. 1-5, 2003.

[12] E. L. Waterworth and J. A. Waterworth, "Focus, Locus, and Sensus: The Three Dimensions of Virtual Experience," Cyberpsychol. Behav., vol. 4, no. 2, pp. 203-213, Apr. 2001, doi: 10.1089/109493101300117893.
[13] G. Riva, J. A. Waterworth, and E. L. Waterworth, "The Layers of Presence: A Bio-cultural Approach to Understanding Presence in Natural and Mediated Environments," Cyberpsychol. Behav., vol. 7, no. 4, pp. 402-416, Aug. 2004, doi: 10.1089/cpb.2004.7.402.

[14] S. Agrawal, A. Simon, and S. Bech, "Defining Immersion: Literature Review and Implications for Research on Immersive Audiovisual Experiences," in 147th AES Pro Audio International Convention, New York, 2019, p. 14.

[15] Committee on How People Learn II: The Science and Practice of Learning, Board on Behavioral, Cognitive, and Sensory Sciences, Board on Science Education, Division of Behavioral and Social Sciences and Education, and National Academies of Sciences, Engineering, and Medicine, How People Learn II: Learners, Contexts, and Cultures. Washington, D.C.: National Academies Press, 2018.

[16] A. Dengel and J. Magdefrau, "Immersive Learning Explored: Subjective and Objective Factors Influencing Learning Outcomes in Immersive Educational Virtual Environments," in 2018 IEEE International Conference on Teaching, Assessment, and Learning for Engineering (TALE), Wollongong, NSW, Dec. 2018, pp. 608-615, doi: 10.1109/TALE.2018.8615281

[17] S. L. Pressey, "A machine for automatic teaching of drill material.," Sch. Soc., 1927.

[18] S. Papert, "Information technology and education: Computer criticism vs. technocentric thinking," Educ. Res., vol. 16, no. 1, pp. 22-30, 1987.

[19] G. Fitzgerald, "Evaluating Information Systems Projects: A Multidimensional Approach,” J. Inf. Technol., vol. 13, no. 1, pp. 15-27, Mar. 1998, doi: 10.1177/026839629801300102.

[20] M. Vaismoradi, J. Jones, H. Turunen, and S. Snelgrove, "Theme development in qualitative content analysis and thematic analysis," $J$. Nurs. Educ. Pract., vol. 6, no. 5, p. p100, Jan. 2016, doi: 10.5430/jnep.v6n5p100.

[21] H. Gaspar, L. Morgado, H. São Mamede, B. Manjón, and C. Gütl, "Identifying immersive environments' most relevant research topics: an instrument to query researchers and practitioners," in iLRN 2018 Montana. Workshop, Long and Short Paper, and Poster Proceedings from the Fourth Immersive Learning Research Network Conference, 2018, pp. 48-71.

[22] M. Akçayır and G. Akçayır, "Advantages and challenges associated with augmented reality for education: A systematic review of the literature," Educ. Res. Rev., vol. 20, pp. 1-11, Feb. 2017, doi: 10.1016/j.edurev.2016.11.002.

[23] J. Bacca, S. Badiris, R. Fabregat, and S. Graf, "Augmented reality trends in education: a systematic review of research and applications," $J$. Ed. Tech. Soc., vol. 17, no. 4, p. 133-149, 2014.

[24] H. Gaspar, L. Morgado, H. Mamede, T. Oliveira, B. Manjón, and C. Gütl, "Research priorities in immersive learning technology: the perspectives of the iLRN community," Virtual Real., vol. 24, pp. 319-341, 2020, doi: 10.1007/s10055-019-00393-x.

[25] M. J. W. Lee, M. Georgieva, B. Alexander, E. Craig, and J. Richter, The State of XR and Immersive Learning Outlook Report: 2020 Edition Executive Summary, Immersive Learning Research Network, Missoula, MT, USA. [Online]. Available: https://immersivelrn.org/wpcontent/uploads/State-of-XR-Outlook-Report-_-Executive-

Summary.pdf. 